\title{
Evaluation of Quality of Life in Patients with Differentiated Thyroid Cancer by Means of the Thyroid-Specific Patient-Reported Outcome Questionnaire: A 5-Year Longitudinal Study
}

\author{
Massimo Giusti ${ }^{a, b}$ Stefano Gay ${ }^{a, b}$ Lucia Conte $^{a, b} \quad$ Francesca Cecoli $^{a}$ \\ Lorenzo Mortara $^{a}$ Lara Vera $^{a}$ Eleonora Monti ${ }^{b}$ \\ ${ }^{a}$ Endocrine Unit, San Martino Polyclinic Hospital, Genoa, Italy; ${ }^{b}$ Department of Internal Medicine, \\ University of Genoa, Genoa, Italy
}

\section{Keywords}

Thyroid cancer · ThyPRO questionnaire · Longitudinal quality of life

\begin{abstract}
Background: Patients with malignancy suffer impairment of their quality of life (QoL). QoL has been evaluated in thyroid cancer patients. Since 2010, a new inventory, the thyroidspecific patient-reported outcome (ThyPRO) measure for benign thyroid disorders, has been available. Aim: This study evaluated QoL longitudinally in patients with a history of differentiated thyroid cancer (DTC) by means of the ThyPRO questionnaire. Methods: From 2012 to 2016, QoL was evaluated yearly in 123 adult DTC patients by means of ThyPRO. The ThyPRO questionnaire consists of 13 scales on which higher scores represent greater impact on QoL in areas affected by thyroid pathology. Disease-specific morbidity due to possible inadequate L-T4 treatment was evaluated by means of the Billewicz scale (BS). The same examinations were conducted in 192 control subjects who had undergone surgery for benign thyroid pathology. Results: DTC and control subjects had similar scores on all but one scale; scores on the hyperthyroid symptoms scale were significantly higher
\end{abstract}

karger@karger.com www.karger.com/etj

(c) 2019 European Thyroid Association Published by S. Karger AG, Basel
Karger ${ }^{\prime}=$ in DTC patients than in controls. Over the 5 years, scores did not change significantly in the DTC group. Overall, QoL and BS scores showed a slight, but not significant, improvement during the study period in DTC patients. BMI impacted on several ThyPRO scales. No significant differences between genders were noted in DTC. Conclusions: The ThyPRO questionnaire indicates that illness perception is similar after thyroidectomy for malign and benign pathology. Only a marginal improvement in QoL was noted in DTC subjects over the 5-year study period. In both groups, females showed a greater perception of illness than males.

(C) 2019 European Thyroid Association Published by S. Karger AG, Basel

\section{Introduction}

In 2003, quality of life (QoL) was defined by Crevenna et al. [1] as "an individual's perception of his or her position in life, in the context of the culture and value systems in which he or she lives, and in relation to his or her goals, expectations, standards and concerns." A diagnosis of cancer causes distress, which can acutely impair QoL. Cancer rehabilitation involves improving psychological 
symptoms and the perception of restored well-being after or during treatment [1]. Moreover, the fear of recurrence is a universal concern in subjects who have had cancer, and persists for a long time after the initial diagnosis, with a substantial effect on QoL [2].

It is well known that differentiated thyroid cancer (DTC) is associated with an excellent medical prognosis, with 10-year survival rates reaching 90-95\% [3]. However, DTC survivors display a high rate of recurrence, and recurrent tumors may develop many years after the initial treatment [4]. Therefore, QoL remains a problem in DTC patients, not only during treatment but also in the long term [5].

To date, most studies have found that DTC patients generally suffer QoL impairment [5-9] and that this is comparable to that of patients with tumors of other sites [10]. For instance, after administering four validated questionnaires to 153 cured DTC patients with a median duration of cure of 6.3 years and a large group of healthy controls matched for age, sex, and socioeconomic status, Hoftijzer et al. [8] documented QoL impairment in the DTC group. Moreover, after a literature search, Singer et al. [9] reported various features of QoL impairment in DTC patients (sudden attacks of tiredness, exhaustion, quality of sleep, employment, social support, fear of cancer progression, fear of second operation, difficulty in swallowing, and global sensation). In the same period, a literature review [11], in which 16 papers regarding QoL in DTC patients were selected, revealed that several factors (surgery, radioiodine therapy, thyroid hormone withdrawal instead of rhTSH administration, access to behavioral help, patient-physician relationship) had both a physical and psychological impact on patients. Finally, Ryu et al. [12] also analyzed the dimension of spiritual well-being, which is not generally explored by other questionnaires, and found that it improved after surgery in patients with DTC of all tumor stages. In 2011, we published a case-controlled longitudinal study on QoL in 128 DTC patients followed up for 5 years, to whom the selfrated Kellner Symptom Questionnaire was administered yearly, in association with a semi-structured psychiatric interview and four other inventories; 219 patients who had undergone surgery for benign thyroid pathology served as a control group [6]. The study documented a wide variation in DTC patients' perception of their illness, and psychological evaluation improved during long-term follow-up, with QoL scores reaching the same levels as in controls. In our opinion, special attention should be devoted to older patients with DTC and to those with advanced staging on diagnosis [6]. Recently,
McIntyre et al. [13] collected data from the a EuroQoL 5-dimension (EQ-5D) questionnaire from 83 cancer patients attending the first UK patient-doctor thyroid cancer forum, and found that the average QoL was lower in DTC patients than in patients with breast, colorectal, or prostate cancer. A large group of these DTC patients suffered from fatigue and depression [13]. These results seem to partially confirm a 2016 report by Applewhite et al. [10], who observed that the self-reported QoL of thyroid cancer survivors was similar to or worse than that of survivors from other types of cancer.

Since 2009, the patient-reported outcome (PRO) measure for benign thyroid disorders (ThyPRO), a specific inventory validated for the assessment of QoL in patients with thyroid disease, has been available in several languages, including Italian [14]. ThyPRO was originally used only in patients with benign thyroid diseases, and thyroid cancer was an exclusion criterion [15]. Subsequently, however, this tool was used by Massolt et al. [16] to assess the association between thyroid function tests and QoL in patients with a history of DTC.

The aim of our 5-year study was to evaluate self-reported QoL in a cohort of DTC subjects by means of the ThyPRO questionnaire. Test scores obtained longitudinally were correlated with clinical data. The objectives of the study were: (a) to apply ThyPRO to patients with malignant thyroid pathology, (b) to confirm by means of ThyPRO our observation of an improvement in QoL over time, and (c) to identify determinants of health-related QoL in DTC patients.

\section{Materials and Methods}

\section{Subjects}

Adult DTC patients under periodic surveillance at the Endocrine Unit of San Martino Polyclinic Hospital were asked to participate in this 5-year longitudinal evaluation of QoL by means of the self-reported ThyPRO questionnaire. In total, 124 subjects (study group) gave their consent to participate in the study, which was conducted during their periodic examinations from 2012 to 2016.

The DTC histology was papillary, follicular variant of papillary, follicular, and other (insular, Hurtle, and cancer of unknown malignant potential) in 102, 9, 6, and 7 subjects, respectively. Retrospective tumor reclassification, according to the 8th edition of the American Joint Committee on Cancer, was available in $99 \%$ of subjects. A total of $82.9 \%$ of DTC were at stage 1 , while the remainder were at stage $2(11.4 \%)$ or stage $3(5.7 \%)$. In the same period, ThyPRO was administered, only once, to 192 consenting adult subjects, who had undergone thyroid surgery for a benign pathology (control group). Table 1 reports the demographic and clinical data of the study and control groups. All but one DTC subject and $91 \%$ of the controls were on treatment with L-T4; all other drugs taken were recorded during each examination. 
Table 1. Some demographic and clinical data of subjects undergoing QoL assessment on entry to the study

\begin{tabular}{lccl}
\hline & $\begin{array}{c}\text { Study } \\
\text { group } \\
(n=124)\end{array}$ & $\begin{array}{l}\text { Control } \\
\text { group } \\
(n=192)\end{array}$ & $p$ value \\
& $59.3 \pm 11.9$ & $60.1 \pm 14.5$ & ns \\
\hline Age, years & $27 / 97$ & $44 / 148$ & ns \\
Male/female ratio & $26.9 \pm 5.0$ & $25.5 \pm 4.3$ & 0.005 \\
BMI & $7.4 \pm 6.8$ & $6.6 \pm 5.1$ & ns \\
Time since surgery, years & & & \\
Thyroidectomy/lobectomy, & $117 / 7$ & $142 / 50$ & $<0.0001$ \\
$\quad$ ratio & 68.5 & 12.5 & $<0.0001$ \\
Previous radio-iodine, \% & $1.68 \pm 0.34$ & $1.31 \pm 0.40$ & $<0.0001$ \\
L-T4 dosages, $\mu$ g/kg body & & & \\
weight/day & $21.2 \pm 4.2$ & $18.2 \pm 3.4$ & $<0.0001$ \\
f-T4, pmol/L & $1.14 \pm 6.87$ & $1.96 \pm 2.28$ & $<0.0001$ \\
TSH, mIU/L & $2.6 \pm 1.8$ & $2.5 \pm 1.9$ & $\mathrm{~ns}$ \\
$\begin{array}{l}\text { Drugs, } n \\
\text { Use of psychotropic drugs, \% }\end{array}$ & 19.4 & 19.3 & $\mathrm{~ns}$ \\
$\begin{array}{l}\text { Major psychopathology, \% } \\
\text { Other cancer, \% }\end{array}$ & 1.6 & 1.0 & $\mathrm{~ns}$ \\
\hline
\end{tabular}

ns, not significant.

\section{Protocol}

At the time of scheduled examinations, all subjects were also asked to complete the self-reported ThyPRO questionnaire. The clinical examination comprised patient history, evaluation of current therapies, physical examination, neck sonography (MyLab Five equipped with a 7.5- to $10.0-\mathrm{MHz}$ linear probe; Esaote, $\mathrm{Ge}$ noa, Italy), and blood tests.

\section{Survey Measurements}

Changes in QoL were measured by means of the Italian version of ThyPRO. This questionnaire consists of 85 questions, grouped into 13 multi-item scales. The ThyPRO scales concern the following aspects: goiter symptoms, hyperthyroid symptoms, hypothyroid symptoms, eye symptoms, tiredness, cognitive problems, anxiety, depression, emotional susceptibility, impaired social life, impaired daily life, impaired sex-life, and cosmetic complaints. Each item is rated on a 5 -point scale from 0 (not at all) to 4 (very much), and refers to the last 4 weeks. Linearly transformed scores vary from 0 to 100 , with higher scores indicating more severe thyroid-related complaints. We also evaluated a further ThyPRO item, dubbed "general score." This regards answers to the question: "In the last 4 weeks has thyroid disease had a negative effect on your QoL?" The "general score" varies from 0 (no impact on QoL) to 4 (very great impact on QoL). The Italian version of the Billewicz scoring index [17], which was converted into an 11-item scale (Billewicz scale; BS) in 1997 [18], was used, as previously [6], in order to assess disease-specific morbidity (or inadequate L-T4 treatment). Seven symptoms (perspiration, hoarseness, paresthesia, skin dryness, constipation, hearing loss, and weight increase) and five signs (motor slowness of movements, lack of Achilles' reflex, rough skin, eyelid edema, and cold skin) were assessed as an observerrated set of clinical findings that have been used in studies of hy- pothyroidism. One point was assigned to each symptom or sign found, with a maximum score of 12. In women under 55 years of age, another point was added to the BS score.

\section{Laboratory Evaluation}

Free-T4 (f-T4) and TSH levels were measured by means of electro-chemiluminescence immunoassay, optimized on the Cobas $\mathrm{E}$ platform (Roche Diagnostics, Milan, Italy); reference ranges are 12.0-22.0 pmol/L for $\mathrm{f}-\mathrm{T} 4$ and $0.3-4.2 \mathrm{mIU} / \mathrm{L}$ for TSH. TSH functional sensitivity is $0.01 \mathrm{mIU} / \mathrm{L}$, with intra- and interassay imprecision of 3 and 7\%, respectively. Thyroglobulin (Tg) and anti-Tg antibodies (TgAb) were evaluated as previously reported [19].

\section{Statistical Analysis}

On the basis of the functional sensitivity of the Tg assays, DTC patients with undetectable Tg levels, negative $\mathrm{TgAb}$, and negative neck sonography were considered disease free. Low-risk DTC patients without a history of radioiodine ablative treatment after surgery in whom Tg levels were low but detectable or stable over time and neck US imaging was negative were also considered to be free from disease. Arbitrarily, the ThyPRO questionnaires were deemed assessable when filled in $\geq 90 \%$ correctly. GraphPad 6.0 software (GraphPad, San Diego, CA, USA) was used for statistical analysis. To compare continuous data, the Kruskal-Wallis analysis of variance (ANOVA), followed by Dunn's multiple comparisons test and the Mann-Whitney test, was used. Percentages were compared by means of the Fisher exact test. Correlations were evaluated by means of the Spearman test. Data are reported as the mean \pm standard deviation (SD) if not otherwise specified. Significance was set at $p \leq 0.05$; exact $p$ values ranging from 0.1 to 0.0001 are given.

\section{Results}

\section{Clinical Data}

Characteristics of the study and control patients are shown in Table 1. At the time of study entry, both groups of subjects were matched for age, male/female ratio, and time since surgical therapy. Because a significantly $(p=$ 0.002 ) greater number of patients were overweight (BMI $>25$ ) or obese (BMI $>30)$ in the study group $(58.1 \%)$ than in the control group (40.1\%), BMI was slightly but significantly higher in DTC patients $(p=0.005)$. Owing to their different diagnoses, the groups differed in terms of therapeutic strategies, L-T4 dosages, and hormonal levels, but there were no differences in the other pharmacological treatments (Table 1). Major psychopathological diseases were diagnosed only in very few subjects. At the time of study entry, a second malignancy was known in some cases, without significant differences between the groups (DTC, 8.8\%; controls, 6.3\%). During the 5-year follow-up period, another 6 malignancies were found in DTC patients, and at the end of the study, the number of known second malignancies was significantly $(p=0.03)$ higher in DTC patients than in controls. 
Fig. 1. Radar plot of mean ThyPRO scores evaluated during the 5-year study period, from year 1 (2012) to year 5 (2016), in the group of DTC patients. The radar plot of mean ThyPRO scores obtained in patients after surgery for benign thyroid pathology (controls) is also shown.

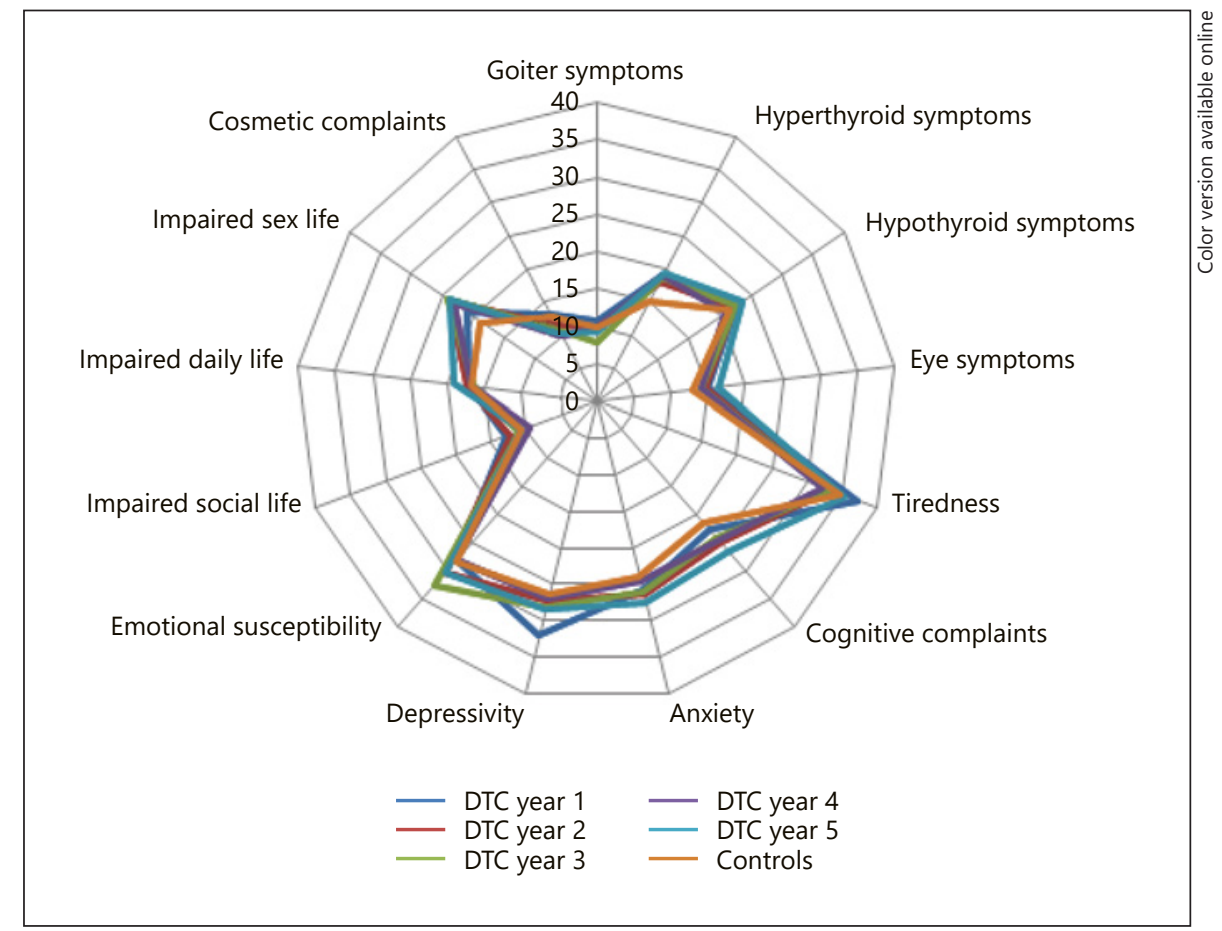

During the 5-year study period, $96 \%$ of DTC patients remained disease free, and the others had stable nonstructural thyroid disease. Owing to the favorable outcome and body weight changes, the L-T4 dosage was significantly $(p=0.02)$ modified during the study period, the average L-T4 dosage being significantly $(p=0.03)$ reduced from the baseline to $1.56 \pm 0.32 \mu \mathrm{g} / \mathrm{kg}$ body weight/ day at the end of the study. No significant change in BMI emerged over the 5-year study period, while drug prescriptions significantly $(p<0.0001)$ increased, owing particularly to the initiation of cholecalciferol and metformin in the DTC group.

\section{Quality of Life}

Figure 1 summarizes the mean ThyPRO scores in the DTC and control groups. Over the 5-year period, no significant variations in scores were noted in the DTC group. Except for the hyperthyroid symptoms scale, no differences emerged between the DTC and control groups. The scores on this scale were lower in controls $(15.1 \pm 13.3)$ than in DTC patients (year 1: $19.0 \pm 15.3, p=0.02$; year 2: $18.8 \pm 15.8, p=0.04$; year $3: 18.7 \pm 15.6, p=0.05$; year 4 : $19.0 \pm 16.7, p=0.05$; year 5: $19.4 \pm 16.6, p=0.03)$. The tiredness scale showed the greatest impairment in thyroid-related QoL in DTC patients. Overall QoL, evaluated by means of the last single item, is reported in Figure
2. This showed a slight, but not significant $(p=0.1)$, improvement in QoL during the study period in DTC patients. At the first evaluation, this score was slightly higher in DTC patients $(0.84 \pm 0.99)$ than in controls $(0.63 \pm$ $0.88, p=0.06)$. BS scores decreased significantly $(p=$ 0.0001 ) over the 5 -year period (Fig. 3). BS scores were slightly higher $(p=0.08)$ in DTC patients than in controls at the beginning of the study but significantly lower $(p=$ 0.04) at the end (Fig. 3).

\section{Determinants of QoL}

TSH levels were unrelated to the majority of ThyPRO scales in both DTC patients and controls on study entry. In DTC patients, the correlation between TSH levels and the goiter symptoms scale was close to significance $(n=$ 118 ; rS 0.18, $p=0.052$ ), while in controls a similar correlation emerged between TSH and the cognitive complaints scale $(n=190 ; \mathrm{rS} 0.14, p=0.06)$. In neither group did a correlation emerge between TSH and BS. In our DTC patients, ThyPRO and BS were unrelated to disease outcome or age on entry to the study, though an inverse correlation between age and cosmetic complaints was close to significance $(n=114 ; \mathrm{rS}-0.18, p=0.06)$. In the study group, a significant male/female difference in ThyPRO scores was seen at the beginning of the study only on the tiredness (males $29.5 \pm 16.5$, females $39.6 \pm 18.6$, 


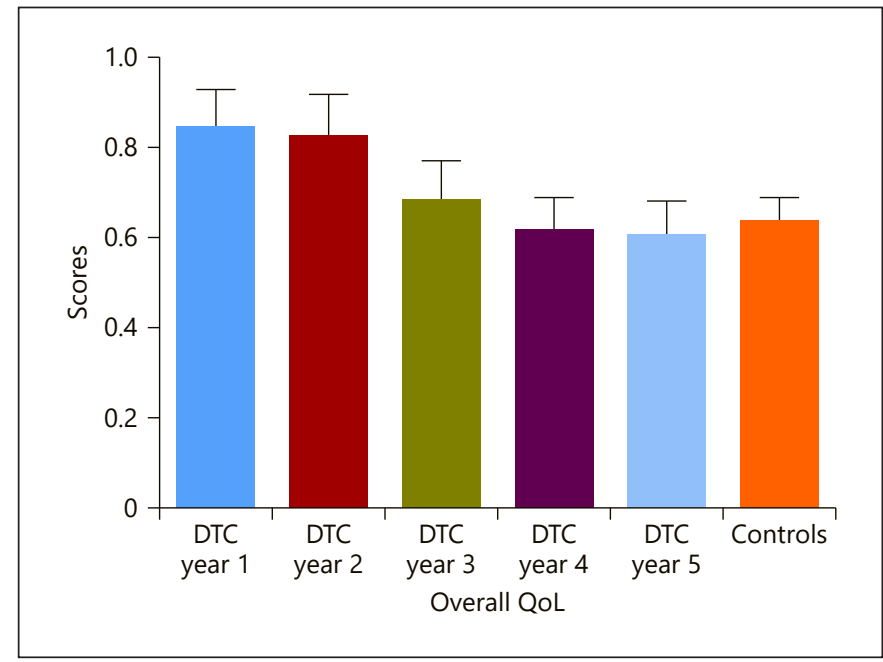

Fig. 2. Mean $( \pm$ SEM) scores of overall QoL evaluated by means of the last single item of ThyPRO during the 5-year study period (from year 1, 2012, to year 5, 2016) in DTC patients and controls.

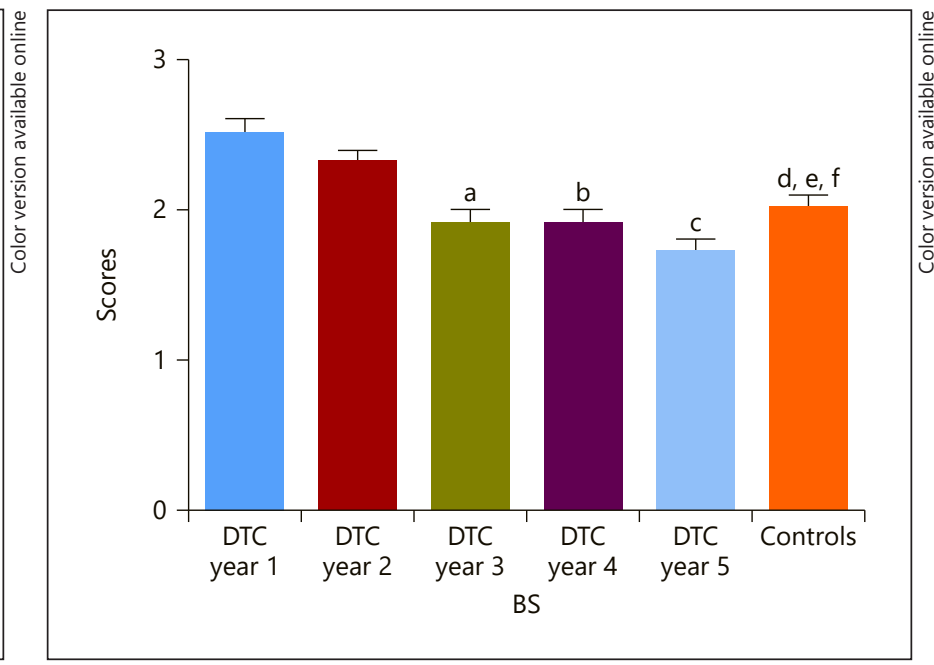

Fig. 3. Mean $( \pm$ SEM) BS scores during the 5 -year study period (from year 1, 2012, to year 5, 2016) in DTC patients and controls. The significance values are: $\mathrm{a}, p=0.05$ versus year 1 ; $\mathrm{b}, p=0.04$ versus year $1 ; c, p=0.0002$ versus year $1 ; \mathrm{d}, p=0.08$ versus year 1 ; e, $p=0.1$ versus year 2 , and $\mathrm{f}, p=0.04$ versus year 5 .
Fig. 4. Radar plot of mean ThyPRO scores evaluated in female and male DTC patients in year 1 (2012). The significance values are: goiter symptoms, $p=0.06$; tiredness, $p=0.02$; depressivity, $p=0.08$, and cosmetic complaints, $p=0.009$.

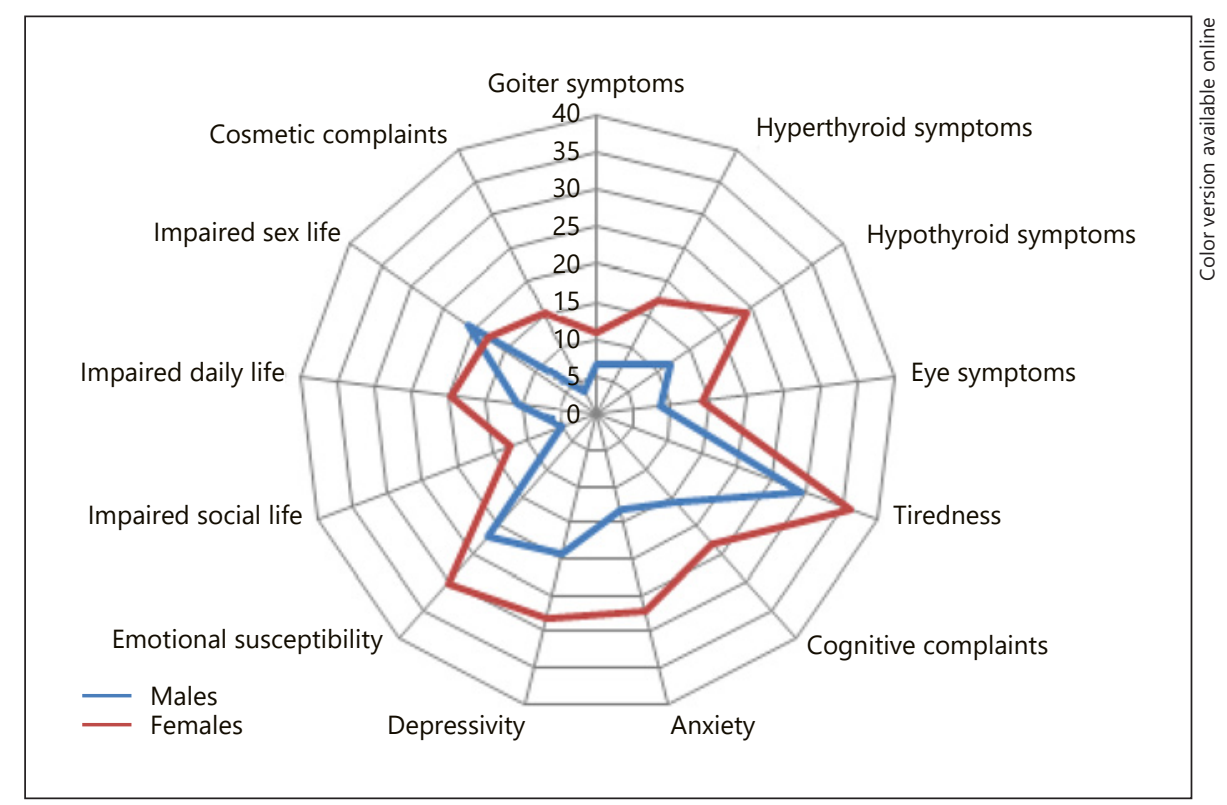

$p=0.02$ ) and cosmetic complaints scales (males $5.1 \pm 9.1$, females $15.2 \pm 19.5, p=0.009$; Fig. 4). Overall QoL, as evaluated by means of the last single item, displayed a nearly significant difference between genders in the control group (males $0.43 \pm 0.76$, females $0.69 \pm 0.91, p=$ 0.051 ) but not in DTC subjects (males $0.67 \pm 0.83$, females $0.91 \pm 1.04)$. BMI had a major impact on ThyPRO scores

Longitudinal ThyPRO Administration in Thyroid Cancer in both groups. In DTC patients, BMI values were positively correlated with the goiter symptoms $(n=118$; rS $0.24, p=0.01)$, hyperthyroid symptoms $(n=115 ; \mathrm{rS} 0.23$, $p=0.01)$, hypothyroid symptoms $(n=114 ; \mathrm{rS} 0.05, p=$ $0.05)$, tiredness $(n=113 ; \mathrm{rS} 0.21, p=0.03)$, and cosmetic complaints $(n=114 ; \mathrm{rS} 0.23, p=0.01)$ scales. In neither group was BMI related to BS. 


\section{Discussion}

It has been reported that an obstacle to evaluating illness perception in patients with thyroid cancer is the lack of specific questionnaires [15], such as those used for thyroid-associated ophthalmopathy [20] and diseasespecific morbidity [7]. In pediatric [21], adolescent [22], and adult DTC patients $[1,5-9,12,16]$, QoL has been evaluated by means of several inventories. Massolt et al. [16] first evaluated QoL in a group of 143 adult DTC patients by means of the ThyPRO questionnaire. In that study, only four ThyPRO scales were reported in detail (hyperthyroid symptoms, tiredness, cognitive complaints, depression) and were investigated in relation to L-T4 dosage, hormonal status, BMI, time since diagnosis, number of drugs taken, and outcome. The authors reported that none of these determinants were associated with scores on the ThyPRO scales evaluated [16]. In 2011, we assessed QoL in 59 DTC patients by means of both the Kellner Symptoms Questionnaire and the new ThyPRO questionnaire [23]. Our preliminary data showed that ThyPRO and the Kellner Symptoms Questionnaire yielded comparable scores in DTC patients and prompted us to employ ThyPRO extensively in these patients [23]. In the present study, longitudinal evaluation of all ThyPRO scales in DTC patients revealed no significant variations in scores over 5 years. Moreover, except for the hyperthyroid symptoms scale, no difference in ThyPRO scores was noted between the DTC and control groups. In both DTC patients and control subjects, the greatest impairment in QoL was seen on the tiredness scale. Indeed, fatigue is a major problem for cancer patients in general [24] and one of the main components of QoL, together with pain [25]. In a study by Singer et al. [7], DTC participants frequently mentioned "sudden attacks of tiredness." However, while tiredness is one of the ThyPRO scales, scores did not seem to be related to hormonal status in our study or in that of Massolt et al. [16]. In addition, in the study by Massolt et al. [16], fatigue was also evaluated by means of the Multidimensional Fatigue Index-20 questionnaire, but did not prove to be associated with thyroid hormone status. Other authors $[8,26]$ found no association between QoL and TSH concentration. Recently, Ryu et al. [12] used the Korean translation of the thyroid-specific QoL scale (KT-QoL) to analyze QoL in a cohort of subjects before and 12 months after thyroid surgery for DTC. Their analysis showed that QoL decreased after surgery, and that QoL impairment was less severe when lobectomy was the surgical choice [12].
The present 5-year study confirmed that the length of time from diagnosis and primary treatments to QoL evaluation plays a role in disease perception. Indeed, in a previous study [26], we observed that disease perception, and consequently QoL, in a subset of 31 DTC patients improved after a psychological examination conducted 8-14 months later. Subsequently, in 128 DTC patients, 5 -year longitudinal evaluation of QoL by means of the self-rating Kellner Symptoms Questionnaire documented changes over time in items including anxiety, somatization, depression, and hostility. Our conclusion was that psychological evaluation during long-term follow-up improved QoL scores, which reached the same levels noted in subjects with a history of thyroid surgery for benign thyroid pathology [6]. In the present study, however, changes in ThyPRO scores over time were less evident in our cohort of 124 DTC patients, and only the hyperthyroid symptoms scale was significantly more impaired in DTC patients than in controls. We believe that yearly medical evaluation in our cohort of DTC patients with a low incidence of biochemical persistence of disease alleviated the negative effects of diagnosis and initial treatment on QoL over time. The persistence of a negative impact on the ThyPRO hyperthyroid symptoms scale may be explained by the relatively high L-T4 dosage maintained in DTC patients. In 2011 [6], we reported that some somatic aspects were less sensitive to yearly examination and we attributed this to an increased perception of symptoms and major comorbidities, such as subclinical thyrotoxicosis, overweight or obesity, and second cancer. In the present study, however, BS scores in DTC patients showed a significantly decreasing trend over the 5-year period, indicating the favorable role of continuing medicalization in DTC patients. In accordance with our results, Nies et al. [21], who studied 67 survivors of pediatric DTC 17.8 years after diagnosis, reported that impaired QoL domains comprising physical problems can be restored only after years of follow-up.

Gender differences in DTC patients' perception of their QoL have been reported. In 2003, Crevenna et al. [1] evaluated 150 consecutive DTC patients by means of the Short Form-36 questionnaire, and found that physical functioning, bodily pain, and role-emotional scales were affected less in males than in females. We previously reported a lower level of somatic symptoms over time in males than in females, but somatization and hostility were significantly lower in DTC females than in DTC males [6]. Nies et al. [21] reported higher levels of reduced motivation and more depression in male survivors of pediatric DTC than in female survivors. In the present 
study, QoL was more severely impaired in females than in males, with significantly higher scores on several of the ThyPRO scales being recorded in females than in males.

In our experience, impaired sex life is the ThyPRO scale that displays the most missing data. This could be explained by the relatively high mean age of the patients, the absence of a partner, the confidential nature of this scale, and the great intrasubject variation in scores during the study period. In this regard, in a study by Rasmussen et al. [27], in which an electronic ThyPRO questionnaire was compared with a paper version in the same population of hyperthyroid patients, the impaired sex life scale also showed the most variability, as indicated by the largest standard deviation. Moreover, in a large sample of patients with benign thyroid pathology whose sex life was explored by means of the ThyPRO questionnaire, Sawicka-Gutaj et al. [28] reported that a third of patients experienced what they perceived as an impaired sex life (women 36\%; men 31\%). A Swiss study [29] examined a small group of 38 couples, one member of which was a DTC patient. Some of the patients and their partners reported that the cancer diagnosis had reduced the frequency of sexual activity. However, compared with cancers of other sites, thyroid cancer had a relatively negative impact on patient-partner relationships [29]. Our data do not clearly indicate that thyroid cancer history, previous therapies, and current hormonal status should be considered major determinants of sex life.

Some studies have used the ThyPRO questionnaire before and after surgery, and it has been reported that ThyPRO scales improve significantly after goiter removal [27, 30-32]. In the study by Massolt et al. [16], the goiter symptoms scale was not commented upon, though in our study, which was not designed to evaluate the effect of surgery, this scale was the one that displayed the lowest scores in all patients at every time point in the study.

The ThyPRO cosmetic complaints scale evaluates how the esthetic outcome of surgery is experienced by the patient. In our study and in that of Rasmussen et al. [27], several surgical factors and individual skin reactivity might explain why scores, albeit not elevated, varied widely among subjects. Surgery followed by lymphadenectomy leaves the patient with a scar which is very difficult to get used to, especially because, for the rest of the patient's life, it remains very exposed, being right in the center of the neck. This could be more important in women, who are often more concerned with physical appearance. Choi et al. [33] evaluated QoL in 97 patients using the Dermatology Life Quality Index. They found that scores did not differ among scar types, but that post-thyroidectomy scars

Longitudinal ThyPRO Administration in Thyroid Cancer on the neck did influence the life of these patients [33]. Dimov [34] reported that neck dissection influenced QoL in DTC patients, especially when post-operative complications were present. Arora et al. [35] evaluated scars in a group of 120 middle-aged subjects, mainly females, after thyroid surgery. Caucasian patients and those with a benign histology reported a lower impact on QoL. The mean scar rating of patients with DTC was shown to be far worse than that of patients with benign disease [35]. We think that the problem of unsightly scars should be considered in DTC patients, and that further improvement in surgery is needed. McIntyre et al. [13] reported that recurrent nerve damage and permanent hypocalcemia were further determinants of QoL impairment in DTC after surgery. In our study, ThyPRO cannot have been influenced by these phenomena, which occurred in less than $3 \%$ of our study and control populations.

Our study has several limitations: (a) as no baseline ThyPRO values were available, information on QoL before surgery was lacking; (b) there are no standard ThyPRO data on the general Italian population; (c) we included very few patients with still active thyroid malignancy (biochemical or structural), and a longitudinal study of this subset of patients by means of the ThyPRO questionnaire is still needed; (d) we did not have sociodemographic data or data on features of sleep - information that is not ordinarily collected, but which could influence ThyPRO responses, and (e) a structured psychiatric interview was not performed.

In conclusion, the ThyPRO questionnaire could be a useful instrument for detecting clinically relevant differences in QoL in DTC, as in benign thyroid pathology. Longitudinal administration of the ThyPRO questionnaire revealed that QoL in thyroid cancer patients a long time after diagnosis and primary treatments is quite similar to that of patients who have undergone surgery for benign thyroid pathology. Only a marginal improvement in QoL was noted in DTC subjects over the 5-year period. ThyPRO is useful in evaluating psychological discomfort in areas not fully explored by other tests. In both groups, females displayed a greater perception of their illness than males.

\section{Acknowledgements}

We thank Luisa Castagnotti for her evaluation of the ThyPRO questionnaires, and all the residents of the Endocrine Unit who collaborated over the years in patient examinations, administration, and the collection of questionnaires. The study was supported by an unrestricted grant from the University of Genoa. 


\section{Statement of Ethics}

Written consent was obtained from all patients. The study protocol was approved by the Ethics Committee of the Department of Internal Medicine of Genoa University. Data collection and subsequent analysis were performed in compliance with the Helsinki Declaration.

\section{Disclosure Statement}

The authors declare no conflicts of interest.

\section{Author Contributions}

Massimo Giusti: study design, data collection, statistical analysis, writing. Stefano Gay, Lucia Conte, Francesca Cecoli, Lorenzo Mortara, Lara Vera: data collection. Eleonora Monti: study design, data collection, writing.

\section{References}

1 Crevenna R, Zettinig G, Keilani M, Posch M, Schmidinger M, Pirich C, et al. Quality of life in patients with non-metastatic differentiated thyroid cancer under thyroxine supplementation therapy. Support Care Cancer. 2003 Sep; 11(9):597-603.

2 van de Wal M, van de Poll-Franse L, Prins J, Gielissen M. Does fear of cancer recurrence differ between cancer types? A study from the population-based PROFILES registry. Psychooncology. 2016 Jul;25(7):772-8.

3 Schlumberger MJ, Torlantano M. Papillary and follicular thyroid carcinoma. Best Pract Res Clin Endocrinol Metab. 2000 Dec;14(4): 601-13.

4 Bilimoria KY, Bentrem DJ, Ko CY, Stewart AK, Winchester DP, Talamonti MS, et al. Extent of surgery affects survival for papillary thyroid cancer. Ann Surg. 2007 Sep;246(3): 375-81.

5 Gamper EM, Wintner LM, Rodrigues M, Buxbaum S, Nilica B, Singer S, et al. Persistent quality of life impairments in differentiated thyroid cancer patients: results from a monitoring programme. Eur J Nucl Med Mol Imaging. $2015 \mathrm{Jul} ; 42(8): 1179-88$.

6 Giusti M, Melle G, Fenocchio M, Mortara L, Cecoli F, Caorsi V, et al. Five-year longitudinal evaluation of quality of life in a cohort of patients with differentiated thyroid carcinoma. J Zhejiang Univ Sci B. 2011 Mar;12(3): $163-73$.

7 Singer S, Husson O, Tomaszewska IM, Locati LD, Kiyota N, Scheidemann-Wesp U, et al. Quality-of-Life Priorities in Patients with Thyroid Cancer: A Multinational European Organisation for Research and Treatment of Cancer Phase I Study. Thyroid. 2016 Nov; 26(11):1605-13.

8 Hoftijzer HC, Heemstra KA, Corssmit EP, van der Klaauw AA, Romijn JA, Smit JW. Quality of life in cured patients with differentiated thyroid carcinoma. J Clin Endocrinol Metab. 2008 Jan;93(1):200-3.

9 Singer S, Lincke T, Gamper E, Bhaskaran K, Schreiber S, Hinz A, et al. Quality of life in patients with thyroid cancer compared with the general population. Thyroid. 2012 Feb; 22(2):117-24
10 Applewhite MK, James BC, Kaplan SP, Angelos P, Kaplan EL, Grogan RH, et al. Quality of Life in Thyroid Cancer is Similar to That of Other Cancers with Worse Survival. World J Surg. 2016 Mar;40(3):551-61.

11 Bãrbus E, Peștean C, Larg MI, Piciu D. Quality of life in thyroid cancer patients: a literature review. Clujul Med. 2017;90(2):147-53.

12 Ryu CH, Park B, Ryu J, Ryu YM, Jo SA, Lee YJ, et al. Development and Evaluation of a Korean Version of a Thyroid-Specific Quality-of-Life Questionnaire Scale in Thyroid Cancer Patients. Cancer Res Treat. 2018 Apr; 50(2):405-15.

13 McIntyre C, Jacques T, Palazzo F, Farnell K, Tolley N. Quality of life in differentiated thyroid cancer. Int J Surg. 2018 Feb;50:133-6.

14 Watt T, Bjorner JB, Groenvold M, Rasmussen AK, Bonnema SJ, Hegedüs L, et al. Establishing construct validity for the thyroid-specific patient reported outcome measure (ThyPRO): an initial examination. Qual Life Res. 2009 May; 18(4):483-96.

15 Watt T, Hegedüs L, Groenvold M, Bjorner JB, Rasmussen AK, Bonnema SJ, et al. Validity and reliability of the novel thyroid-specific quality of life questionnaire, ThyPRO. Eur J Endocrinol. 2010 Jan;162(1):161-7.

16 Massolt ET, van der Windt M, Korevaar TI, Kam BL, Burger JW, Franssen GJ, et al. Thyroid hormone and its metabolites in relation to quality of life in patients treated for differentiated thyroid cancer. Clin Endocrinol (Oxf). 2016 Nov;85(5):781-8.

17 Billewicz WZ, Chapman RS, Crooks J, Day ME, Gossage J, Wayne E, et al. Statistical methods applied to the diagnosis of hypothyroidism. Q J Med. 1969 Apr;38(150):255-66.

18 Zulewski H, Müller B, Exer P, Miserez AR, Staub JJ. Estimation of tissue hypothyroidism by a new clinical score: evaluation of patients with various grades of hypothyroidism and controls. J Clin Endocrinol Metab. 1997 Mar; 82(3):771-6.

19 Giusti M, Mortara L, Machello N, Monti E, Pera G, Marenzana M. Utility of a liquid formulation of levo-thyroxine in differentiated thyroid cancer patients. Drug Res (Stuttg). 2015 Jun;65(6):332-6.
20 Terwee CB, Dekker FW, Mourits MP, Gerding MN, Baldeschi L, Kalmann R, et al. Interpretation and validity of changes in scores on the Graves' ophthalmopathy quality of life questionnaire (GO-QOL) after different treatments. Clin Endocrinol (Oxf). 2001 Mar; 54(3):391-8.

21 Nies M, Klein Hesselink MS, Huizinga GA, Sulkers E, Brouwers AH, Burgerhof JG, et al. Long-Term Quality of Life in Adult Survivors of Pediatric Differentiated Thyroid Carcinoma. J Clin Endocrinol Metab. 2017 Apr; 102(4):1218-26.

22 Oren A, Benoit MA, Murphy A, Schulte F, Hamilton J. Quality of life and anxiety in adolescents with differentiated thyroid cancer. J Clin Endocrinol Metab. 2012 Oct; 97(10):E1933-7.

23 Pera G, Gallo F, Mortara L, Cecoli F, Giusti M. Valutazione della qualità della vita (QoL) nel paziente con carcinoma differenziato della tiroide (CDT): comparazione tra Kellner Symptoms Questionnaire e questionario ThyPRO. Proceedings of the 5th National Congress of the Italian Thyroid Association; 2011 Dec 1-3. Padua: Grafiche SP; 2011. Abstract, p. 134.

24 Kuhnt S, Ernst J, Singer S, Rüffer JU, Kortmann RD, Stolzenburg JU, et al. Fatigue in cancer survivors-prevalence and correlates. Onkologie. 2009 Jun;32(6):312-7.

25 Costantini M, Mencaglia E, Giulio PD, Cortesi E, Roila F, Ballatori E, et al. Cancer patients as 'experts' in defining quality of life domains. A multicentre survey by the Italian Group for the Evaluation of Outcomes in Oncology (IGEO). Qual Life Res. 2000 Mar;9(2):151-9.

26 Giusti M, Sibilla F, Cappi C, Dellepiane M, Tombesi F, Ceresola E, et al. A case-controlled study on the quality of life in a cohort of patients with history of differentiated thyroid carcinoma. J Endocrinol Invest. 2005 JulAug;28(7):599-608.

27 Rasmussen SL, Rejnmark L, Ebbehøj E, FeldtRasmussen U, Rasmussen ÅK, Bjorner JB, et al. High Level of Agreement between Electronic and Paper Mode of Administration of a Thyroid-Specific Patient-Reported Outcome, ThyPRO. Eur Thyroid J. 2016 Mar; 5(1):65-72. 
28 Sawicka-Gutaj N, Ruchala M, Feldt-Rasmussen U, Rasmussen ÅK, Hegedüs L, Bonnema SJ, et al. Patients with Benign Thyroid Diseases Experience an Impaired Sex Life. Thyroid. 2018 Oct;28(10):1261-9.

29 Büel-Drabe N, Steinert H, Moergeli H, Weidt S, Seiler A, Jenewein J. Thyroid cancer has a small impact on patient-partner relationships and their frequency of sexual activity. Palliat Support Care. 2018 Jun;16(3):335-46.

30 Sorensen JR, Watt T, Cramon P, Døssing H, Hegedüs L, Bonnema SJ, et al. Quality of life after thyroidectomy in patients with nontoxic nodular goiter: A prospective cohort study. Head Neck. 2017 Nov;39(11):2232-40.
31 Sorensen JR, Lauridsen JF, Døssing H, Nguyen N, Hegedüs L, Bonnema SJ, et al. Thyroidectomy Improves Tracheal Anatomy and Airflow in Patients with Nodular Goiter: A Prospective Cohort Study. Eur Thyroid J. 2017 Nov;6(6):307-14.

32 Mishra A, Sabaretnam M, Chand G, Agarwal G, Agarwal A, Verma AK, et al. Quality of life (QoL) in patients with benign thyroid goiters (pre- and post-thyroidectomy): a prospective study. World J Surg. 2013 Oct;37(10):2322-9.
33 Choi Y, Lee JH, Kim YH, Lee YS, Chang HS, Park CS, et al. Impact of postthyroidectomy scar on the quality of life of thyroid cancer patients. Ann Dermatol. 2014 Dec;26(6):6939.

34 Dimov RS. The effect of neck dissection on quality of life in patients with differentiated thyroid cancer. Gland Surg. 2013 Nov;2(4): 219-26.

35 Arora A, Swords C, Garas G, Chaidas K, Prichard A, Budge J, et al. The perception of scar cosmesis following thyroid and parathyroid surgery: a prospective cohort study. Int $J$ Surg. 2016 Jan;25:38-43. 